\title{
Linear response and collective oscillations in superconductors with $d$-wave pairing
}

\author{
S.N. Artemenko, A.G. Kobelkov \\ Institute for Radioengineering and Electronics of the Russian Academy of Sciences, 103907 \\ Moscow, Russia
}

(June 23, 2021)

\begin{abstract}
Simple equations for the linear response of layered superconductors with $d-$ wave symmetry of the order parameter are derived by means of kinetic equations for Green's functions. Responses to solenoidal and potential electric fields have different frequency dependencies. The damping of plasma oscillations of superconducting electrons is determined by dielectric relaxation and is small. Relaxation of branch imbalance determined by elastic scattering is large enough to make the Carlson-Goldman mode in $d$-wave superconductors overdamped.
\end{abstract}

74.25.Fy, 74.25.N1, 74.20.Mn

Typeset using REVTEX 
Many evidences for $d$-wave symmetry (or near $d$-wave symmetry) of the superconducting order parameter in layered high $-\mathrm{T}_{c}$ superconductors were given last years by measurements of the Josephson effect [1] [3], by microwave experiments [4], by high-resolution angle-resolved spectroscopy [5] and by other methods. On the other hand, theoretical studies confirm a compatibility of many experimental data with $d$-wave symmetry of the superconducting gap in high- $\mathrm{T}_{c}$ superconductors (see [6,8] and references therein). Many properties of the $d$-wave superconductors are expected to be different ifrom those of conventional superconductors, especially the effects related to quasiparticles and their relaxation, since due to the nodes of the $d$-wave order parameter the quasiparticle density is never exponentially small. Here, we study theoretically the effects related to electric field, and collective oscillations in $d$-wave superconductors.

Typically, calculations of the linear response of the superconductors assume the response to a solenoidal (transverse) electric field which can be expressed in terms of time derivative of the vector potential, paying less attention to a potential (longitudinal) electric field which is determined as the gradient of the scalar potential. However, the linear response of superconductors depends on the origin of the electric field. Electric field created by variations of the current density and magnetic field in time, and related to the Faraday's law, creates in a superconductor only the perturbations of the electronic distribution with the antisymmetric angle dependence. Such a perturbation is limited by the momentum relaxation, like in a normal metal. On the other hand, the potential electric field which is related to perturbations of the charge density and to the Coulomb's law creates, in addition, the branch imbalance [8.9.11], $i$. e. the difference between the densities of electron-like and hole-like quasiparticles. Thus, relaxation of the branch imbalance is involved in the linear response of a superconductor to the potential electric field as well. The branch imbalance is characterized by the gauge invariant scalar potential $\mu=(1 / 2)(\partial \chi / \partial t)+\Phi$ where $\chi$ is the phase of the order parameter and $\Phi$ is the electric potential. Potential $\mu$ can be interpreted as the nonequilibrium shift of the chemical potential of the normal carreers. Furthermore, the superconducting momentum, $\mathbf{P}_{s}=(1 / 2) \nabla \chi-\mathbf{A}$ where $\mathbf{A}$ is the vector potential, plays 
a role of the gauge invariant vector potential (see [1] and references therein). Then the electric field is expressed in terms of the gauge invariant potentials as

$$
\mathbf{E}=-\nabla \mu+\frac{\partial \mathbf{P}_{s}}{\partial t} .
$$

So, two contributions to the electric field in (11) produce different kinds of the perturbations of the electronic distribution in a superconductor. We shall consider the first term of this expression as the potential part of the electric field. The second term related to the variations of the superconducting current in time we shall call the solenoidal part of the electric field. Strictly speaking, the second term in (11) does not satisfy the usual definition of the solenoidal field because its divergence does not vanish exactly in all the cases. Nevertheless, we call it solenoidal or transverse field because it is needed to describe the purely solenoidal field, and it is related to the response to the electric field created by the time dependent magnetic field.

The potential electric field must be taken into account in the problem of the linear response since it is important in collective oscillations and it appears in nonuniform and anisotropic systems, even if externally applied field is purely solenoidal. In order to study the problem we calculate a linear response of $d$-wave superconductors to the gauge invariant vector and scalar electromagnetic potentials, and derive simple and physically transparent expressions for the charge and current densities from the equations for quasiclassical Green's functions using the nonequilibrium approach by Keldysh [10]. Such an approach enables us to take into account both momentum and branch imbalance relaxation rates. The latter enters the generalized conductivity describing the response to the potential electric field. Then we use these expressions to study plasma oscillations of superconducting electrons and the Carlson-Goldman mode in $d$-wave superconductors.

To calculate the linear response of layered superconductors with $d$-wave pairing, we start with the equations for Green's functions in Keldysh technique, using the slightly modified approach by Larkin and Ovchinnikov [12]. We use two different ways to describe layered superconductors. In the first approach we use a continuous representation, considering an 
anisotropic metal with the band motion of electrons in the direction perpendicular to the layers such that $t_{\perp} \gg \nu$, where $t_{\perp}$ is the overlap integral describing the electron spectrum in perpendicular direction, $\epsilon_{\perp}=2 t_{\perp} \cos d p_{\perp}$. Here $d$ is the lattice constant in the perpendicular direction, and $\nu$ the momentum scattering rate along the layers. In the second approach we use similar equations [13],[14] for layered superconductors in the discrete Wannier representation, considering the hopping conductivity regime between the layers, i. e. $t_{\perp} \ll \nu$, which corresponds to the case of Josephson interlayer coupling. The second approach bears some similarity to the interlayer diffusion model [15] in which the interlayer coupling is mediated through incoherent hopping processes with $t_{\perp}$ neglected. In both cases we assume a $d$-wave superconducting order parameter: thus we do not address the question of the microscopic nature of the interaction resulting in such a symmetry.

To derive the equations in the continuous representation we subtract, similar to [12], from the equation for matrix Green's function in Keldysh representation its conjugated equation. Then we integrate the resulting equation over $\xi=p_{\|}^{2} / 2 m-\varepsilon_{F}$, where $\mathbf{p}_{\|}$is the component of the momentum parallel to the layers. Thus we obtain equations for the retarded (advanced) Green's functions, $g^{R(A)}$, and for $g^{K}$, which is related to the electron distribution function. Each of these functions is a matrix in Nambu space and depends on coordinates, energies, perpendicular component $p_{\perp}$ of the momentum, and on the angle $\phi$ of $\mathbf{p}_{\|}$.

In the linear approximation with respect to the external perturbation, the equation for the anomalous Green's function, $g^{(a)}$, defined by $g^{K}=g^{R}\left(\varepsilon, \varepsilon^{\prime}\right) \tanh \left(\varepsilon^{\prime} / 2 T\right)-$ $g^{A}\left(\varepsilon, \varepsilon^{\prime}\right) \tanh (\varepsilon / 2 T)+g^{(a)}\left(\varepsilon, \varepsilon^{\prime}\right)$ has the form

$$
\begin{array}{r}
\mathbf{v} \nabla g^{(a)}-\left[\varepsilon_{+} \sigma_{z}+\Delta(\phi) i \sigma_{y}\right] g^{(a)}+g^{(a)}\left[\varepsilon_{-} \sigma_{z}+\Delta(\phi) i \sigma_{y}\right]- \\
\left(\Sigma^{R} g^{(a)}-g^{R} \Sigma^{(a)}+\Sigma^{(a)} g^{A}-g^{(a)} \Sigma^{A}\right)=\alpha\left[\left(\mathbf{v} \mathbf{P}_{s} \sigma_{z}+\mu\right) g^{A}-g^{R}\left(\mathbf{v} \mathbf{P}_{s} \sigma_{z}+\mu\right)\right] .
\end{array}
$$

Here $\mathbf{v}$ is the electron velocity at the Fermi surface, $\Delta(\phi)$ is the amplitude of the order parameter, $\alpha=\tanh \varepsilon_{+} / 2 T-\tanh \varepsilon_{-} / 2 T, \sigma_{y, z}$ are Pauli matrices, and the unperturbed retarded and advanced Green's functions, $g^{R(A)}$, depend on shifted energies $\varepsilon_{+}=\varepsilon+\omega / 2$ and $\varepsilon_{-}=\varepsilon-\omega / 2$, respectively. The self-energy parts, $\Sigma^{\iota}$, are given by 


$$
\hat{\Sigma}^{\iota}=\int_{-\pi / d}^{\pi / d} \frac{d p_{\perp}^{\prime}}{2 \pi / d} \int_{0}^{2 \pi} \frac{d \phi^{\prime}}{2 \pi} \nu\left(p_{\perp}, \phi ; p_{\perp}^{\prime}, \phi^{\prime}\right) \hat{g}^{\iota}\left(p_{\perp}^{\prime}, \phi^{\prime}\right),
$$

where $\iota=\mathrm{R}, \mathrm{A}$, or $\mathrm{K} ; \nu$ is the elastic scattering rate in the normal state. Strictly speaking, (3) describes the impurity scattering in Born approximation, but it can be applied also to elastic scattering by phonons, since the related self-energy part acquires the form (3) when one neglects phonon frequencies in comparison with electron energies in delta-functions describing the energy conservation law in scattering processes. Using Born approximation we neglect low-energy quasiparticle bound states created by impurities (see [16] and references therein), and, hence, our results are applicable provided typical energies of quasiparticles are larger than the bandwidth of the impurity induced bound states, $T>\sqrt{\Delta \nu}$.

Now we consider the momentum dependence of the scattering rate $\nu$. As it will be seen below, the in-plane scattering results in pair-breaking similar to magnetic impurities in swave superconductors, while the interlayer scattering does not affect the gap. In addition, from the conductivity anisotropy data in high- $\mathrm{T}_{c}$ superconductors one may expect, that corresponding components of $\nu$ have different temperature dependencies. Having this in mind we consider a simple model for momentum dependence of $\nu$, which takes into account different scattering rates in different directions:

$$
\nu\left(p_{\perp}, \phi ; p_{\perp}^{\prime}, \phi^{\prime}\right)=\nu_{i}+\nu_{\perp} \delta\left(\phi-\phi^{\prime}\right)
$$

Here $\nu_{i}$ describes the isotropic scattering, and $\nu_{\perp}$ is related to the scattering in the perpendicular, interlayer, direction.

Using (3) and (4) we obtain for the unperturbed retarded (advanced) Green's functions in (2) the implicit relations $g^{R(A)}=\sigma_{z} a^{R(A)}+i \sigma_{y} b^{R(A)}$, where $a^{R(A)}=\left(\varepsilon+i \nu_{i}\left\langle a^{R(A)}\right\rangle_{\phi} / 2\right) / \xi^{R(A)}$, $b^{R(A)}=\Delta(\phi) / \xi^{R(A)}$. The brackets $\langle\ldots\rangle$ mean averaging over variables mentioned in the subscript, and $\xi^{R(A)}= \pm \sqrt{\left(\varepsilon+i \nu_{i}\left\langle a^{R(A)}\right\rangle_{\phi} / 2\right)^{2}-\Delta(\phi)^{2}}$. The equations for perturbations of $g^{R(A)}$ can be obtained from (2) by replacing $\alpha$ by 1 , changing all the superscripts for $R(A)$, and omitting the two last terms in the collision integral.

The main differences in the equations for the Green's functions in the discrete representation (see [14]) are the following. Green's functions become matrices in layer indices, and 
the potentials depend on the layer index as well. The first term in the l.h.s. of (2) in the discrete representation is to be replaced by $t_{\perp} \sum_{i= \pm 1}\left(A_{n n+i} g_{n+i m}^{(a)}-g_{n m+i}^{(a)} A_{m+i m}\right)$, describing the interlayer interaction, with $A_{n m}=\cos \left(\chi_{n}-\chi_{m}\right) / 2+i \sigma_{z} \sin \left(\chi_{n}-\chi_{m}\right) / 2$. Averaging in the collision integral is performed over the angle $\phi$ only.

We solve the linearized equations for Green's functions for the case of smoothly varying perturbations $|\mathbf{q v}| \ll \nu_{i}, i$. e. when changes of all variables along the distance of the order of the mean free path along the layers are small. This case covers the most interesting range of frequencies, because characteristic values of $1 / q$ are determined either by the magnetic penetration lengths (at low frequencies) which are typically larger, than the mean free path in high- $\mathrm{T}_{c}$ superconductors, or (at high frequencies) by the skin-effect length, which is also large provided the frequency is below the range of the anomalous skin-effect.

Perturbations of charge density are determined by $\operatorname{Tr}\left\langle g^{(a)}\right\rangle$ integrated over energies. Quasiparticle current densities in the directions parallel and perpendicular to the conducting layers are proportional to integrals of $\operatorname{Tr} \sigma_{z}\left\langle\mathbf{v} g^{(a)}\right\rangle$ and of $\operatorname{Tr} \sigma_{z}\left\langle v_{z} g^{(a)}\right\rangle$, respectively. Superconducting currents are determined by similar terms with retarded and andvanced Green's functions in the relation between $g^{K}$ and $g^{(a)}$. We calculate current and charge densities assuming the clean limit, $T_{c} \gg \nu_{i}$, since in the opposite dirty limit a superconductor is in a gapless state. In the case of frequencies $\omega$ much smaller than the amplitude of the gap, $\Delta$ the linear response can be presented in a simple and physically transparent form:

$$
\begin{array}{r}
-i \omega \rho=-i \omega \gamma \frac{\kappa^{2}}{4 \pi} \mu+\left(\sigma_{2 l} q^{2}+\sigma_{2 t} k^{2}\right) \mu+\omega\left(\sigma_{1 l} \mathbf{q} \mathbf{P}_{l}+\sigma_{1 t} k P_{t}\right), \\
\mathbf{j}_{l}=\frac{c^{2}}{4 \pi \lambda_{l}^{2}} \mathbf{P}_{l}-i\left(\omega \sigma_{0 l} \mathbf{P}_{l}+\sigma_{1 l} \mathbf{q} \mu\right), \\
j_{t}=\frac{c^{2}}{4 \pi \lambda_{t}^{2}} P_{t}-i\left(\omega \sigma_{0 t} P_{t}+\sigma_{1 t} k \mu\right),
\end{array}
$$

where $\mathbf{P}_{l}$ and $P_{t}$ are parallel and perpendicular to the layers components of $\mathbf{P}_{s}, \mathbf{q}$ and $k$ are parallel and perpendicular components of wave vector, $\kappa^{-1}$ is the Thomas-Fermi screening radius, and $\lambda_{l(t)}$ are the penetration lengths for a superconducting current parallel (perpendicular) to the layers. 
The first terms in (6, (6) describe the supercurrents, while the last terms are related to the quasiparticles. Taking into account that the electric field expressed in terms of the gauge invariant potentials is given by (11) we see, that the simple expression $\mathbf{j}=\hat{\sigma} \mathbf{E}$ for quasiparticle contributions to the currents is not valid: generalized conductivities $\sigma_{n \alpha}$ are different for the contributions from scalar and vector potentials to the electric field. This implies different responses to the potential and to the solenoidal electric fields. Then note that according to (5) the changes of the charge density are determined by time variations of the potential $\mu$, which is related to the electrone-hole imbalance (cf. [9, 11]), and by space variations of the quasiparticle currents. Equation (5) plays a role of the continuity equation for normal carreers.

The factor $\gamma$ and the generalized conductivities $\sigma_{n \alpha}(n=0,1,2$ and $\alpha=l, t)$ depend on frequency:

$$
\begin{array}{r}
\gamma=1+\int_{-\infty}^{\infty} d \varepsilon \frac{\omega\left\langle a_{0}\right\rangle_{\phi}}{\left(\omega+i \nu_{b}\right)} \frac{d n_{F}}{d \varepsilon} \\
\sigma_{n \alpha}=-\sigma_{N \alpha} \frac{1}{\tau_{\alpha}} \int_{-\infty}^{\infty} d \varepsilon\left\langle\frac{i \theta(|\varepsilon|-|\Delta(\phi)|) a_{0}^{1-2 n} \omega^{n}}{\left(\omega+i \tilde{\nu}_{\alpha}\right)\left(\omega+i \nu_{b}\right)^{n}} \frac{d n_{F}}{d \varepsilon}\right\rangle_{\phi}
\end{array}
$$

Here $\sigma_{N \alpha}$ is the normal state conductivity in direction $\alpha, n_{F}$ is Fermi distribution function, and $a_{0}=\varepsilon / \sqrt{\varepsilon^{2}-\Delta^{2}} ; \tau_{l} \equiv 1 / \nu_{i}=1 / \nu_{l}$ and $\tau_{t}=1 /\left(\nu_{i}+\nu_{\perp}\right)$ are the momentum scattering times of electrons in the normal state for longitudinal and transverse directions, and $\tilde{\nu}_{l}=$ $\nu_{l}\left\langle a_{0}\right\rangle_{\phi}$ and $\tilde{\nu}_{t}=\tilde{\nu}_{l}+\nu_{\perp} / a_{0}$ are energy dependent effective scattering rates of quasiparticles for corresponding directions. Finally, $\nu_{b}=\nu_{l}\left\langle\Delta^{2}(\phi) a_{0} / \varepsilon^{2}\right\rangle_{\phi}$ is the effective branch imbalance relaxation rate. It is well-known that in s-wave superconductors the branch imbalance relaxes via inelastic scattering, spin-flip scattering or due to anisotropy of the order parameter (for a review see [11]). In the case of $d$-wave pairing the elastic scattering is a main source of the branch imbalance relaxation.

In the studies of the linear response to a solenoidal field, the conductivity $\sigma_{0 l}$ in (6) is usually calculated. Our result for $\sigma_{0 l}$ agrees with the Born limit of the general expressions for the conductivity obtained in [7].

Note that the conductivities in the transverse direction are determined by contributions 
both from intralayer scattering and by interlayer scattering, so that the effective scattering rate for the conductivity in transverse direction is larger than the effective scattering rate for the in-plane quasiparticle current.

Solution of the discrete equations in the limit of small phase differences between the neighbouring layers gives results similar to (5-9); it can be obtained from (5-7) substituting $P_{t}$ for $\left(\chi_{n}-\chi_{n-1}\right) / d$, and $v_{z}$ for $2 t_{\perp} d$ with $\nu_{\perp}=0$ and $\nu_{i} \equiv \nu$ in (8-9), so that conductivities in both directions are determined by the same scattering rate.

Now we discuss the limits of low $(\Delta \gg T)$ and high $(\Delta \ll T)$ temperatures.

1. $\Delta \gg T$ : an important distinction from s-wave superconductors is that the conductivities in (9) are not exponentially small.

Consider, first, the linear response to the electromagnetic wave. For simplicity, in order to get explicit expressions we consider the simplest angular dependence of the gap parameter with the $d$-wave symmetry $\Delta=\Delta_{0} \cos 2 \phi$. At low temperatures, $\left\langle a_{0}\right\rangle_{\phi}=\varepsilon / \Delta_{0}$, and the characteristic times for quasiparticles averaged over energies are $\tilde{\tau}_{l}=\tau_{l}\left(\Delta_{0} / 2 T\right) \approx$ $\tau_{b} \gg \tau_{l}$. The relative density of superconducting component in $d$-wave superconductor is $N_{s}=1-\left(T / \Delta_{0}\right) \ln 4=(\lambda(0) / \lambda(T))^{2} \approx 1$. The factor $\gamma$ is also very close to $1, \gamma=1+2 i \omega \tau_{l}$ for $\omega \ll 1 / \tau_{b}$, and $\gamma=1-\left(T / \Delta_{0}\right) \ln 4$ for $\omega \gg 1 / \tau_{b}$. This leads to a smaller contribution of scalar potential $\mu$ (though not exponentially small as in s-wave superconductors), and we may omit the diffusion contribution to the quasiparticle current densities. Then the current densities can be presented as

$$
\begin{array}{r}
\mathbf{j}_{\alpha}=\frac{c^{2}}{4 \pi \lambda_{\alpha}^{2}} \mathbf{P}_{\alpha}-i \omega \sigma_{N \alpha} \mathbf{P}_{\alpha} R_{\alpha}(\omega) \\
R_{l}=\int_{0}^{\infty} \frac{x d x}{\left(x-i \omega \tilde{\tau}_{l}\right) \cosh ^{2} x} \\
R_{t}=\frac{2 \tau_{l}}{\pi \tau_{t}} \int_{0}^{\infty} d x \int_{0}^{1} \frac{x d y}{\left(x-i \omega \tilde{\tau}_{l}+\nu_{\perp} \tilde{\tau}_{l} \sqrt{1-y^{2}}\right) \cosh ^{2} x}
\end{array}
$$

According to (10-11), at $\omega \tilde{\tau}_{l} \ll 1, i$. e. when scattering is important, the decrease of the normal carreer density is compensated by the decrease of the scattering rate of quasiparticles in comparison with the normal state by the same factor $\propto T / \Delta$. Furthermore, $R_{l}=1$, and 
the quasiparticle conductivity along the layers even at low temperatures is the same as it would be in the normal state at this temperature. At higher frequencies $\left(\omega \tilde{\tau}_{l} \gg 1\right)$ $R_{l}=i\left(1-N_{s}\right) /\left(\omega \tau_{l}\right)$, scattering is not important and the current density corresponds to a free motion of all electrons.

Consider now the conductivity in the transverse direction. Note that due to the depairing action of the in-plane elastic scattering, these processes contribute to the conductivity in the transverse direction. If $\nu_{\perp} \ll 1 / \tilde{\tau}_{l}$ or $\nu_{\perp} \ll \omega$, we find $R_{t}=R_{l}\left(\tau_{l} / \tau_{t}\right)$. Thus in this case the transverse conductivity is determined by the in-plane scattering. At $\nu_{\perp} \gg 1 / \tilde{\tau}_{l}$ and $\nu_{\perp} \gg \omega$, we obtain $R_{t} \propto\left(T / \Delta_{0}\right) \ln \nu_{\perp}^{2} /\left(\omega^{2}+1 / \tilde{\tau}_{l}^{2}\right)$ is small and can be neglected.

Using equations (10-12) one can easily calculate the surface impedance of a $d$-wave superconductor. For a surface parallel to the layers we obtain

$$
\zeta=\frac{\omega \lambda_{l}}{c} \sqrt{\frac{1-\left(\omega / \omega_{0}\right)^{2}-i R_{t} \omega \tau_{t}+\left(q \lambda_{t}\right)^{2}}{\left(1-\left(\omega / \omega_{0}\right)^{2}-i R_{t} \omega \tau_{t}\right)\left(1-i R_{l} \omega \tau_{l}\right)}}
$$

where $\omega_{0}=c / \lambda_{t}$ is the frequency of plasma oscillations for an electric field perpendicular to the layers.

We conclude that, in spite of the large quasiparticle conductivity equal to the normalstate conductivity, the damping terms at low temperatures are always small, because the scattering of quasiparticles is important only at $\omega<1 / \tilde{\tau}_{l} \ll 1 / \tau_{l}$.

Now we discuss the spectrum of free oscillations, which can be calculated inserting (5(7) into the Maxwell equations. The spectrum of the weakly damped plasma mode in the long-wavelength limit is given by an expression similar to that of the case of $s$-pairing [14]:

$$
\omega^{2}=\omega_{0}^{2}\left(\frac{1+k^{2} \lambda_{l}^{2}+q^{2} \lambda_{t}^{2}}{1+k^{2} \lambda_{l}^{2}}-i R_{t} \omega \tau_{t}\right)
$$

The last term in (14) describes damping. For small frequencies limit it is determined by the dielectric relaxation frequency $4 \pi \sigma_{N}$, which is rather large. Nevertheless, the plasma oscillations survive, because the damping is determined by $\sigma_{N}$ only at frequencies $\omega<$ $T /(\tau \Delta) \ll 1 / \tau$, and becomes small at $\omega \approx \omega_{0}>T /(\tau \Delta)$. 
2. $\Delta \ll T$ : at high temperatures (but outside the gapless regime $\Delta<\nu_{l}$ ) the branch imbalance relaxation rate is much smaller than the elastic collision rate, and the conductivities (9) depend on the relation between frequency $\omega$ and $\nu_{l}(\Delta / T)^{2}$.

In the frequency range $\omega \gg \nu_{l}(\Delta / T)^{2}$ in $s$-wave superconductors, where the factor $\gamma$ is real (see [1]), the weakly damped Carlson-Goldman mode appears. In $d$-wave superconductors the factor $\gamma=\left(\pi \Delta_{0} / 2 T\right) \sqrt{\left.i \nu_{l} / \omega\right)}$ contains a large imaginary part due to the larger imbalance relaxation rate, and the related mode is highly damped.

In the static limit our equations determine the penetration length $l_{E}$ of the electric field into a $d$-wave superconductor in direction $\alpha$, when a current flows through a contact with a normal metal. Very near $\mathrm{T}_{c}$ when one can neglect Andreev reflection of the quasiparticles we obtain $l_{E}=\sqrt{\left(\pi \Delta_{0} D_{\alpha} \tau_{l}\right) /(4 T)}$, which agrees with the results of Choi [17]. Here $D_{\alpha}$ are diffusion coefficients related to the conductivities $\sigma_{N \alpha}$ by the relation $D_{\alpha} \kappa^{2}=4 \pi \sigma_{N \alpha}$. The anisotropy of $l_{E}^{2}$ is proportional to the conductivity anisotropy.

If the order parameter is not of the pure $d$-type symmetry, but is close to it: $\langle\Delta(\phi)\rangle^{2} \ll$ $\left\langle\Delta(\phi)^{2}\right\rangle$, then the results of our calculations are qualitatively the same. The main distinctions appear in the different energy and angle dependencies of the quasiparticle relaxation times.

In conclusion, we calculated the linear response of layered $d$-wave superconductors by means of the kinetic theory. We found the conductivities determining the quasiparticle currents created by the longitudinal and by the transverse electromagnetic fields. These results were applied to describe collective modes and the decay length of the electric field near the boundary with a normal metal.

We are indebted to U. Eckern for reading the manuscript and helpful comments. 


\section{REFERENCES}

[1] D. A. Wollman et al., Phys. Rev. Lett. 71, 2134 (1993).

[2] I. Iguchi and Z. Wen, Phys. Rev. B 49, 12388 (1994).

[3] D. A. Browner and H. R. Ott, Phys. Rev. B 53, 8249 (1996).

[4] W. N. Hardi et al., Phys. Rev. Lett. 70, 399 (1993).

[5] T. Yokoya et al., Phys. Rev. B 53, 14055 (1996).

[6] M. Sigrist and T. M. Rice, J. Phys. Soc. Jpn. 61, 4283 (1992).

[7] P. J. Hirschfeld, W. O. Putikka, and D. J. Scalapino, Phys. Rev. Lett. 71, 3705 (1993); Phys. Rev. B 50, 10250 (1996).

[8] M. Tinkham, J.Clarke, Phys. Rev. Lett. 28, 1366 (1972).

[9] M. Tinkham, Phys. Rev. B 6, 1747 (1972).

[10] L. V. Keldysh, Zh. Eksp. Teor. Fiz. 47, 1515 (1964) [Sov. Phys. JETP 20, 1018 (1977)].

[11] S. N. Artemenko, A. F. Volkov, Uspekhi Fiz. Nauk 128, 3 (1979) [Sov. Phys. Usp. 22, $295(1979)]$

[12] A. I. Larkin, Yu. N. Ovchinnikov, Zh. Eksp. Teor. Fiz. 73, 299 (1977) [Sov. Phys. JETP 46, 155 (1977)].

[13] S. N. Artemenko, Zh. Eksp. Teor. Fiz. 79, 162 (1980) [Sov. Phys. JETP 52, 81 (1980)].

[14] S. N. Artemenko, A. G. Kobel'kov, JETP Letters. 58, 445 (1993); Physica C 253, 373 $(1995)$

[15] M. Graf, D. Rainer, and J. Sauls, Phys. Rev. B 47, 12089 (1993).

[16] M. Graf et al., Phys. Rev. B 53, 15147 (1996).

[17] C. H. Choi, Phys. Rev. B 54, 3044 (1996). 